\title{
Epidemiological Characteristics Of Central Nervous System Tumors In Children: A 5-Year Review of 3,180 Cases From Beijing Tiantan Hospital
}

\author{
Zhiming Liu \\ Capital Medical University \\ Chihyi Liao \\ Capital Medical University \\ Heng Zhang \\ Capital Medical University \\ Zhe Han \\ Capital Medical University \\ Junmei Wang \\ Capital Medical University

\section{Zhenyu Ma} \\ Capital Medical University \\ Chunde Li \\ Capital Medical University \\ Jian Gong \\ Capital Medical University \\ Wei Liu \\ Capital Medical University \\ Tao Sun \\ Capital Medical University \\ Yongji Tian ( $\nabla$ tianyongji@bjtth.org ) \\ Beijing Tiantan Hospital https://orcid.org/0000-0001-9915-8630
}

\section{Research}

Keywords: Epidemiology, Central Nervous System tumors, Pediatric, World Health Organization classification

Posted Date: July 14th, 2021

DOI: https://doi.org/10.21203/rs.3.rs-693878/v1

License: (c) (i) This work is licensed under a Creative Commons Attribution 4.0 International License. Read Full License 


\section{Abstract}

Objective: To describe the epidemiological characteristics of central nervous system tumors in children, based on the neurosurgery department of Beijing Tiantan Hospital.

Methods: From January 2015 to December 2019, 3180 children were histopathologically diagnosed with central nervous system tumors based on the 2016 World Health Organization classification of tumors. Patients were 0 to 15 years old. We analyzed age-related gender preferences, tumor locations and the histological grades of the tumors. In addition, the epidemiological characteristics of the five most common intracranial tumors were compared to the previous studies.

Results: In this study, intracranial and spinal tumors account for $96.4 \%$ (3 066) and 3.6\% (114) of all tumors, with a preponderance of supratentorial tumors (57.9\%). Among all pediatric patients, low-grade tumors comprise $67.1 \%(2$ 135). The integral gender ratio of males to females is $1.47: 1$ and the average age of patients is 7.59 years old. The five most common intracranial tumors are craniopharyngioma (15.4\%), medulloblastoma (14.3\%), pilocytic astrocytoma $(11.8 \%)$, diffuse astrocytoma (9.8\%), and anaplastic ependymoma (4.8\%).

Conclusions: Due to lack of national data on childhood brain tumors, we used a large nationally representative population sample based on the largest pediatric neurosurgery center in China. We analyzed the data of the past five years, reflecting the incidence of CNS tumors in Chinese children to a certain extent, and laying a data foundation for subsequent clinical studies.

\section{Introduction}

Neurological neoplasms account for the majority of solid tumors in children and are the primary cause of malignancyrelated deaths. In recent decades, advances in genetics and molecular biology have led to improvements in the diagnosis and treatment methods in pediatric neuro-oncology around the world. The latest World Health Organization (WHO) classification of tumors of the central nervous system has also improved the criteria for diagnosis ${ }^{1}$. China is a developing country with the largest population in the world, and there are many pediatric patients with neurological neoplasms. With the rapid increase and mobility in the population, we need to update the latest data. As National Brain Tumor Registry of China is still under construction, we collected the latest data from Beijing Tiantan Hospital Pediatric Neurosurgery Department (China's largest Pediatric Neurosurgery Center $^{2}$ ), hoping to understand the population distribution characteristics of CNS tumors in children so as to lay a foundation for the follow-up clinical research.

\section{Materials And Methods}

From January 2015 to December 2019, 3180 pediatric patients up to 15 years old underwent surgery to remove primary neurological neoplasms in Beijing Tiantan Hospital. The patients came from more than 30 provinces, municipalities and autonomous regions in China. In this retrospective study, all patients were diagnosed histopathologically by the same group of pathologists from the Department of Pathology in Beijing Tiantan Hospital across the 5-year period. The histological diagnosis and tumor grade were assessed according to the 2016 World Health Organization classification of central nervous system tumors ${ }^{1}$. WHO grade I and grade II indicate low-grade tumors, and WHO grade III and IV indicate high-grade tumors. Supratentorial tumors consist of tumors of the cerebral hemisphere, third ventricle and lateral ventricle, saddle region and pineal region; infratentorial tumors include tumors of the cerebellum, brainstem and fourth ventricle.

All patients were divided artificially into five age groups of 2-year intervals except infant group (0-3 years of age): 4-6, 7$9,10-12$, and 13-15 years of age. For each patient with tumor recurrence whose pathological typing and/or grade changed, we remained the initial diagnosis to standardize the results. In terms of the latest classification, we adjusted some items in this study. For example, we included fibrillary astrocytoma as diffuse astrocytoma because their diagnoses are 
almost exactly the same, and gliomatosis cerebri was deleted as a distinct entity ${ }^{1,3}$. Due to the difficulty of popularizing molecular testing in developing countries, the diagnosis of all tumors was still based on morphologic features.

\section{Results}

In 3,180 children with central nervous system tumors, the prevalence of intracranial tumors was $96.4 \%(3,066)$, while that of spinal tumors was $3.6 \%$ (114). The sex ratio of males to females was 1.47:1. The majority of all patients had low-grade tumors, which accounted $66.4 \%$ (2 036) in brain and 86.8\% (99) in spinal cord. For intracranial tumor location, supratentorial (57.9\%) tumors were slightly more preponderant than infratentorial tumors (42.1\%). The percentages of affected male patients, low-grade CNS tumors, and supratentorial tumor location are shown for each age group, the intracranial tumor group and the spinal tumor group (shown in Fig. 1). Based on the new WHO classification of CNS tumors, we listed the prevalence of each major group of tumors (shown in Fig. 2). Diffuse astrocytic and oligodendroglial tumors $(19.34 \%)$ accounted for the majority of tumors, followed by embryonal tumors $(16.37 \%)$ and tumors of the sellar region (15.39\%). Due to the large population of affected children, we observed diversity in the histopathology of neurological neoplasms and differences between intracranial and spinal tumors (shown in Fig. 3).

The five most frequent intracranial tumors were craniopharyngioma (15.4\%), medulloblastoma (14.3\%), pilocytic astrocytoma (11.8\%), diffuse astrocytoma (9.8\%), and anaplastic ependymoma (4.8\%). These five tumors accounted for $56.1 \%$ of all intracranial tumors (1 721/3 066), and each tumor was more common in males than in females. The highest male-female ratio was observed in anaplastic ependymoma (2.08:1), followed by medulloblastoma (1.89:1). Regarding the tumor locations, the majority were supratentorial tumors, and the ratio of supratentorial tumors to infratentorial tumors was 1.37:1. However, in addition to medulloblastoma and craniopharyngioma (both located in the infratentorial or supratentorial region), pilocytic astrocytoma, diffuse astrocytoma and anaplastic ependymoma were all more commonly found in the infratentorial region (shown in Table 1). It is worth noting that there were only 37 cases of germinomas in this study, which does not indicate a sharp reduction in the incidence rate but rather a more effective and less harmful treatment strategy.

Table 1

Age-related frequency, sex ratio, histological and topographical distribution of the 5 most common intracranial tumors [n, $\mathrm{n}(\%)]$. ( $\mathrm{M}=$ Male; $\mathrm{F}=$ female; $\mathrm{IT}=$ infratentorial; $\mathrm{ST}=$ supratentorial $)$

\begin{tabular}{|c|c|c|c|c|c|c|c|c|}
\hline \multirow[t]{2}{*}{ Histological types } & \multirow[t]{2}{*}{ Frequency } & \multirow{2}{*}{$\begin{array}{l}0-3 \\
\text { years }\end{array}$} & \multirow{2}{*}{$\begin{array}{l}4-6 \\
\text { years }\end{array}$} & \multirow{2}{*}{$\begin{array}{l}7-9 \\
\text { years }\end{array}$} & \multirow{2}{*}{$\begin{array}{l}10-12 \\
\text { years }\end{array}$} & \multirow{2}{*}{$\begin{array}{l}13-15 \\
\text { years }\end{array}$} & \multicolumn{2}{|l|}{ Ratios } \\
\hline & & & & & & & M:F & IT:ST \\
\hline Craniopharyngioma & $472(15.4)$ & $70(2.3)$ & $149(4.9)$ & $109(3.6)$ & $88(2.9)$ & $56(1.8)$ & $1.37: 1$ & - \\
\hline Medulloblastoma & $437(14.3)$ & $57(1.9)$ & $136(4.4)$ & $137(4.5)$ & $73(2.4)$ & $34(1.1)$ & $1.89: 1$ & - \\
\hline $\begin{array}{l}\text { Pilocytic } \\
\text { astrocytoma }\end{array}$ & $362(11.8)$ & $77(2.5)$ & $110(3.6)$ & $75(2.4)$ & $58(1.9)$ & $42(1.4)$ & $1.14: 1$ & $1.59: 1$ \\
\hline $\begin{array}{l}\text { Diffuse } \\
\text { astrocytoma }\end{array}$ & $302(9.8)$ & $72(2.3)$ & $81(2.6)$ & $59(1.9)$ & $54(1.8)$ & $36(1.2)$ & $1.32: 1$ & $1.56: 1$ \\
\hline $\begin{array}{l}\text { Anaplastic } \\
\text { ependymoma }\end{array}$ & $148(4.8)$ & $45(1.5)$ & $54(1.8)$ & $29(0.9)$ & $12(0.4)$ & $8(0.3)$ & $2.08: 1$ & $1.60: 1$ \\
\hline Intracranial tumors & $3,066(100)$ & $556(18.1)$ & $818(26.7)$ & $687(22.4)$ & $553(18.0)$ & $452(14.8)$ & $1.47: 1$ & 0.73:1 \\
\hline
\end{tabular}

Concerning the distribution of age across the common intracranial tumors, in all age groups, the prevalences of children diagnosed with medulloblastoma between 4-6 years old and 7-9 years old were almost the same. However, the other common intracranial tumors were high prevalent among children in the 4- 6 age group. Medulloblastoma was most common in male children (286 cases), while craniopharyngioma was most common in female children (199 cases). Unlike intracranial tumors, the most common spinal tumor was perineurioma (20 cases), followed by lipoma (13 cases), 
ependymoma (9 cases), pilocytic astrocytoma (8 cases) and mature teratoma (8 cases). The prevalence of spinal tumors increased with age. There were slightly more males with spinal tumors than females, and the ratio was 1.28:1.

Although our study is based on a single center, 3,180 pediatric patients came from more than 30 provincial administrative units. Shandong Province and Hebei Province had the most children with CNS tumors, with 470 (14.8\%) and 460 (14.5\%) cases, respectively, followed by Henan Province (10.3\%), Anhui Province (6.3\%) and Shanxi Province (4.7\%). The data showed that the time distributions of CNS tumors from various regions was different. The majority of pediatric patients in 2015, 2017 and 2019 came from Hebei Province, while most patients in 2016 and 2018 came from Shandong Province (shown in Table 2).

Table 2

Province and year distribution of childhood central nervous system tumors [n, $n$ $(\%)]$.

\begin{tabular}{|lllllll|}
\hline Provice & $\mathbf{n}(\%)$ & $\mathbf{2 0 1 5 y}$ & $\mathbf{2 0 1 6 y}$ & $\mathbf{2 0 1 7 y}$ & $\mathbf{2 0 1 8 y}$ & $\mathbf{2 0 1 9 y}$ \\
\hline Shandong & $470(14.8)$ & $87(2.7)$ & $97(3.1)$ & $104(3.3)$ & $102(3.2)$ & $80(2.5)$ \\
\hline Hebei & $460(14.5)$ & $88(2.8)$ & $82(2.6)$ & $108(3.4)$ & $85(2.7)$ & $97(3.1)$ \\
\hline Henan & $326(10.3)$ & $79(2.5)$ & $70(2.2)$ & $68(2.1)$ & $58(1.8)$ & $51(1.6)$ \\
\hline Anhui & $200(6.3)$ & $42(1.3)$ & $42(1.3)$ & $46(1.4)$ & $34(1.1)$ & $36(1.1)$ \\
\hline Shanxi & $148(4.7)$ & $34(1.1)$ & $31(1.0)$ & $36(1.1)$ & $26(0.8)$ & $21(0.7)$ \\
\hline
\end{tabular}

\section{Discussion}

From 2012 to 2016, the average annual incidence of primary CNS tumors for patients 19 years of age was 6.06 cases per 100000 people in the United States ${ }^{4}$. Lower incidence rates were reported in other parts of the world, such as Japan (3.61 per 100000 children $)^{5}$, Germany $(2.6)^{6}$, Italy $(3.46)^{7}$, and Taiwan $(1.7)^{8}$. The reported incidence rate of CNS tumors increased slightly for those $0-19$ years of age over the past 30 years in the US ${ }^{4,9,10}$. This increase in the incidence is partly due to the improvements in diagnostic techniques, including magnetic resonance imaging (MRI), which has significantly enhanced the sensitivity of diagnosis ${ }^{11-13}$. Therefore, these findings illustrated the importance of the disease surveillance and registry system ${ }^{11,14}$, and reflected the demand for a registry system for CNS tumors among children (based on populations or hospitals) in China. Previous studies showed that pediatric intracranial tumors were more common in males $^{15-18}$. We found a 1.47:1 male-to-female ratio, indicating that male children were more likely to be affected, similar to previous literature reports. This predominance among male children was most evident in anaplastic ependymoma and medulloblastoma. However, there was a slightly higher incidence in female children in our study than in the study conducted in Beijing Tiantan Hospital from 2001 to $2005^{15}$.

Most of the pathological tumor types described in the latest WHO classification were observed in this retrospective study, demonstrating the diversity of CNS tumors among children. Studies have reported that the pathological types of intracranial tumors in children are different from those in adults. Meningiomas, pituitary tumors and malignant gliomas are the most common types of adult intracranial tumors ${ }^{19}$, whereas our study showed that craniopharyngioma was the most frequent type of intracranial tumor in children. Common intracranial tumors also included medulloblastoma, pilocytic astrocytoma, diffuse astrocytoma, and anaplastic ependymoma; the prevalence of these tumors herein was slightly different than those reported other countries ${ }^{4,6,7}$. The most notable aspect of this study is the prevalence of GCTs, mostly observed in pediatric patients in Asia20,21. However, during the period from 2015 to 2019, only 37 children were diagnosed with pure germinomas (1.16\%) and treated with surgical resection. These data do not indicate a significant reduction in the prevalence of germinomas but rather improvements in the diagnosis and treatment of these tumors over the last two decades. Germinomas are extremely sensitive to radiotherapy, and a complete response could be achieved with 
radiotherapy alone in most cases 22,23 ; thus, pediatric patients with germinoma are more likely to avoid surgery except in emergency situations.

The overall data regarding age and tumor location showed that tumors were more commonly found in the supratentorial region than the infratentorial region in each age group. The largest difference was observed in the 13-15 years old group. However, the most common intracranial tumors are found in the infratentorial region (except craniopharyngioma, which originates only in the sellar region). In the group aged 7-9 years old, infratentorial tumors accounted for the highest proportion, which may be due to the high incidence of medulloblastoma. An analysis of tumor grade and children's age showed that the majority of high-grade and low-grade tumors were found in children aged 4-6 years old, and the majority of tumors in each age group were low-grade. This illustrated that the peak age of children with intracranial tumors is younger than in previous studies ${ }^{15}$.

Regarding province and year distribution in childhood intracranial tumors, although the patients were from almost all of the country's provincial districts, they were not distributed evenly. Shandong Province and Hebei Province had the most pediatric patients, while Hong Kong and the Tibet Autonomous Region had the fewest patients. These results were related to the distance from Beijing, the level of medical care and the economic level in the location of the patients. The overall trend over the 5-year period was not obvious. The number of patients was the largest in 2017, while there was a small decline in 2019. This trend may be related to the continuous development of medical criteria in various regions over recent years.

The research suggested that the central nervous system tumors is still one of the most important factors affecting children's lives. Differences in age, region, and ethnicity may be potential factors affecting the pathological type and incidence rate of intracranial tumors in children ${ }^{4,10}$. Among the top five most common types of intracranial tumors in this research, the majority were low-grade tumors, and the ratio of low-grade tumors to high-grade tumors was 1.94:1 (1 136/585). Among these intracranial tumors, craniopharyngioma, pilocytic astrocytoma and diffuse astrocytoma are all lowgrade tumors. This finding was related to the polymorphism of children's tumor genes and the location of tumor origins ${ }^{24}$. Due to the popularization of neuroimaging technologies ${ }^{13,24}$, children with intracranial brain tumors can be diagnosed and treated earlier and more precisely; however, the prognosis and quality of life of children still need to be further analyzed.

Based on these findings, we are looking forward to multicenter and multidisciplinary collaborative research. Combining domestic regional characteristics to establish the disease surveillance and registry system and to improve epidemiological follow-up practices will be significant for the diagnosis and treatment of intracranial tumors among children in China.

\section{Conclusion}

First, the complete follow-up and registry system in China is still under construction, so not all cases in our study have follow-up results. And second, molecular genotyping has gradually gained popularity recently, but it has not been analyzed in the early cases because of the restriction of cost and time. Thus, we could not completely keep to the latest WHO classification. Beijing Tiantan Hospital has the largest pediatric neurosurgery center in China ${ }^{2}$, although it cannot represent the national data, we believe that it is still of necessary research significance, in order to establish a national follow-up and registry system in the future. In summary, more in-depth researches by providing an overview of epidemiological

information on primary brain tumors were needed to promote multidisciplinary research ${ }^{24}$.

\section{Declarations}

\section{Ethics approval and consent to participate}


All procedures performed in studies involving human participants with the ethical standards of the institutional and/or national research committee by the 1964 Helsinki Declaration and its later amendments or comparable ethical standards. This study was approved by Institutional Review Board of Beijing Tiantan Hospital, Capital Medical University. Approval reference number is 'KY 2020-090-02'. All experiments were performed in accordance with relevant named guidelines and regulations and we didn't take any interventions in this retrospective study. The subjects in the study are under 18 and informed consent was obtained from legal guardians of all subjects.

\section{Consent for publication}

Written informed consent for publication was obtained from all participants.

\section{Availability of data and materials}

The datasets generated during and/or analysed during the current study are available from the corresponding author on reasonable request.

\section{Competing interests}

All authors declared no competing interests.

\section{Funding}

This research was not funded by individuals or institutions.

\section{Authors' Contributions}

Zhiming Liu: Conceptualization, Methodology, Validation, Data Curation, Formal analysis, Writing - Original Draft

Chihyi Liao: Conceptualization, Validation, Data Curation, Writing - Review \& Editing

Heng Zhang: Conceptualization, Validation, Data Curation, Writing -Review \& Editing

Zhe Han: Conceptualization, Validation, Data Curation, Writing - Review \& Editing

Junmei Wang: Conceptualization, Validation, Data Curation

Zhenyu Ma: Conceptualization, Supervision, Writing - Review \& Editing

Chunde Li: Conceptualization, Supervision, Writing - Review \& Editing

Jian Gong: Conceptualization, Supervision, Writing - Review \& Editing

Wei Liu: Conceptualization, Writing - Review \& Editing

Tao Sun: Conceptualization, Writing - Review \& Editing

Yongji Tian: Conceptualization, Methodology, Validation, Data Curation, Formal analysis, Writing - Review \& Editing, Project administration

\section{Acknowledgements}

In writing this paper, I have benefited from the presence of my roommates. They generously helped me collect materials I needed and made many invaluable suggestions. I hereby extend my grateful thanks to them for their kind help, without which the paper would not have been what it is. 


\section{References}

1. Louis DN, et al. The 2016 World Health Organization Classification of Tumors of the Central Nervous System: a summary. Acta Neuropathol. 2016;131:803-20. doi:10.1007/s00401-016-1545-1.

2. Liu W, Tang J, Van Halm-Lutterodt N, Luo S, Li C. History and current state of pediatric neurosurgery at Beijing Tiantan Hospital Neurosurgery Center. Childs Nerv Syst. 2018;34:797-803. doi:10.1007/s00381-018-3755-4.

3. Chhabda S, Carney O, D'Arco F, Jacques TS, Mankad K. The 2016 World Health Organization Classification of tumours of the Central Nervous System: what the paediatric neuroradiologist needs to know. Quant Imaging Med Surg. 2016;6:486-9. doi:10.21037/qims.2016.10.01.

4. Ostrom QT, et al. CBTRUS Statistical Report: Primary Brain and Other Central Nervous System Tumors Diagnosed in the United States in 2012-2016. Neuro Oncol. 2019;21:v1-100. doi:10.1093/neuonc/noz150.

5. Makino K, Nakamura H, Yano S, Kuratsu J. \& Kumamoto Brain Tumor, G. Population-based epidemiological study of primary intracranial tumors in childhood. Childs Nerv Syst. 2010;26:1029-34. doi:10.1007/s00381-010-1126-x.

6. Kaatsch, P., Rickert, C. H., Kuhl, J., Schuz, J. \& Michaelis, J. Population-based epidemiologic data on brain tumors in German children. Cancer 92, 3155-3164, doi:10.1002/1097-0142(20011215)92:12<3155::aid-cncr10158>3.0.co;2-c (2001).

7. Group AW, Ccm \& Group AW. Italian cancer figures, report 2012: Cancer in children and adolescents. Epidemiol Prev. 2013;37:1-225.

8. Liu YL, et al. Incidence of cancer in children aged 0-14 years in Taiwan, 1996-2010. Cancer Epidemiol. 2015;39:21-8. doi:10.1016/j.canep.2014.11.010.

9. Levy AS. Brain tumors in children: evaluation and management. Curr Probl Pediatr Adolesc Health Care. 2005;35:23045. doi:10.1016/j.cppeds.2005.04.001.

10. Ostrom QT, et al. CBTRUS Statistical Report: Primary Brain and Central Nervous System Tumors Diagnosed in the United States in 2008-2012. Neuro Oncol. 2015;17 Suppl(4):iv1-62. doi:10.1093/neuonc/nov189.

11. Stiller CA, Nectoux J. International Incidence of Childhood Brain and Spinal Tumors. Int J Epidemiol. 1994;23:458-64. doi:DOI 10.1093/ije/23.3.458.

12. Legler JM, et al. Cancer surveillance series [corrected]: brain and other central nervous system cancers: recent trends in incidence and mortality. J Natl Cancer Inst. 1999;91:1382-90. doi:10.1093/jnci/91.16.1382.

13. Sklar CA. Childhood brain tumors. J Pediatr Endocrinol Metab. 2002;15(Suppl 2):669-73. doi:10.1515/jpem.2002.15.s2.669.

14. Smith, M. A., Freidlin, B., Ries, L. A. G. \& Simon, R. Increased incidence rates but no space-time clustering of childhood astrocytoma in Sweden, 1973-1992 - A population-based study of pediatric brain tumors. Cancer 88, 1492-1493, doi:Doi 10.1002/(Sici)1097 - 0142(20000315)88:6 < 1492::Aid-Cncr30 > 3.0.Co;2-7 (2000).

15. Zhou D, et al. Epidemiology of nervous system tumors in children: a survey of 1,485 cases in Beijing Tiantan Hospital from 2001 to 2005. Pediatr Neurosurg. 2008;44:97-103. doi:10.1159/000113110.

16. Ostrom QT, et al. Alex's Lemonade Stand Foundation Infant and Childhood Primary Brain and Central Nervous System Tumors Diagnosed in the United States in 2007-2011. Neuro Oncol. 2015;16 Suppl(10):x1-36. doi:10.1093/neuonc/nou327.

17. Linabery AM, Ross JA. Trends in childhood cancer incidence in the U.S. (1992-2004). Cancer. 2008;112:416-32. doi:10.1002/cncr.23169.

18. Rickert $\mathrm{CH}$, Paulus W. Epidemiology of central nervous system tumors in childhood and adolescence based on the new WHO classification. Childs Nerv Syst. 2001;17:503-11. doi:10.1007/s003810100496.

19. McNeill KA. Epidemiology of Brain Tumors. Neurol Clin. 2016;34:981-98. doi:10.1016/j.ncl.2016.06.014. 
20. Jennings MT, Gelman R, Hochberg F. Intracranial germ-cell tumors: natural history and pathogenesis. J Neurosurg. 1985;63:155-67. doi:10.3171/jns.1985.63.2.0155.

21. Hoffman HJ, et al. Intracranial germ-cell tumors in children. J Neurosurg. 1991;74:545-51.

doi:10.3171/jns.1991.74.4.0545.

22. Matsutani $\mathrm{M}$, et al. Primary intracranial germ cell tumors: a clinical analysis of 153 histologically verified cases. J Neurosurg. 1997;86:446-55. doi:10.3171/jns.1997.86.3.0446.

23. Sawamura Y, Ikeda J, Shirato H, Tada M, Abe H. Germ cell tumours of the central nervous system: treatment consideration based on 111 cases and their long-term clinical outcomes. Eur J Cancer. 1998;34:104-10. doi:10.1016/s0959-8049(97)10045-4.

24. Pollack IF. Multidisciplinary management of childhood brain tumors: a review of outcomes, recent advances, and challenges. J Neurosurg Pediatr. 2011;8:135-48. doi:10.3171/2011.5.PEDS1178.

\section{Figures}

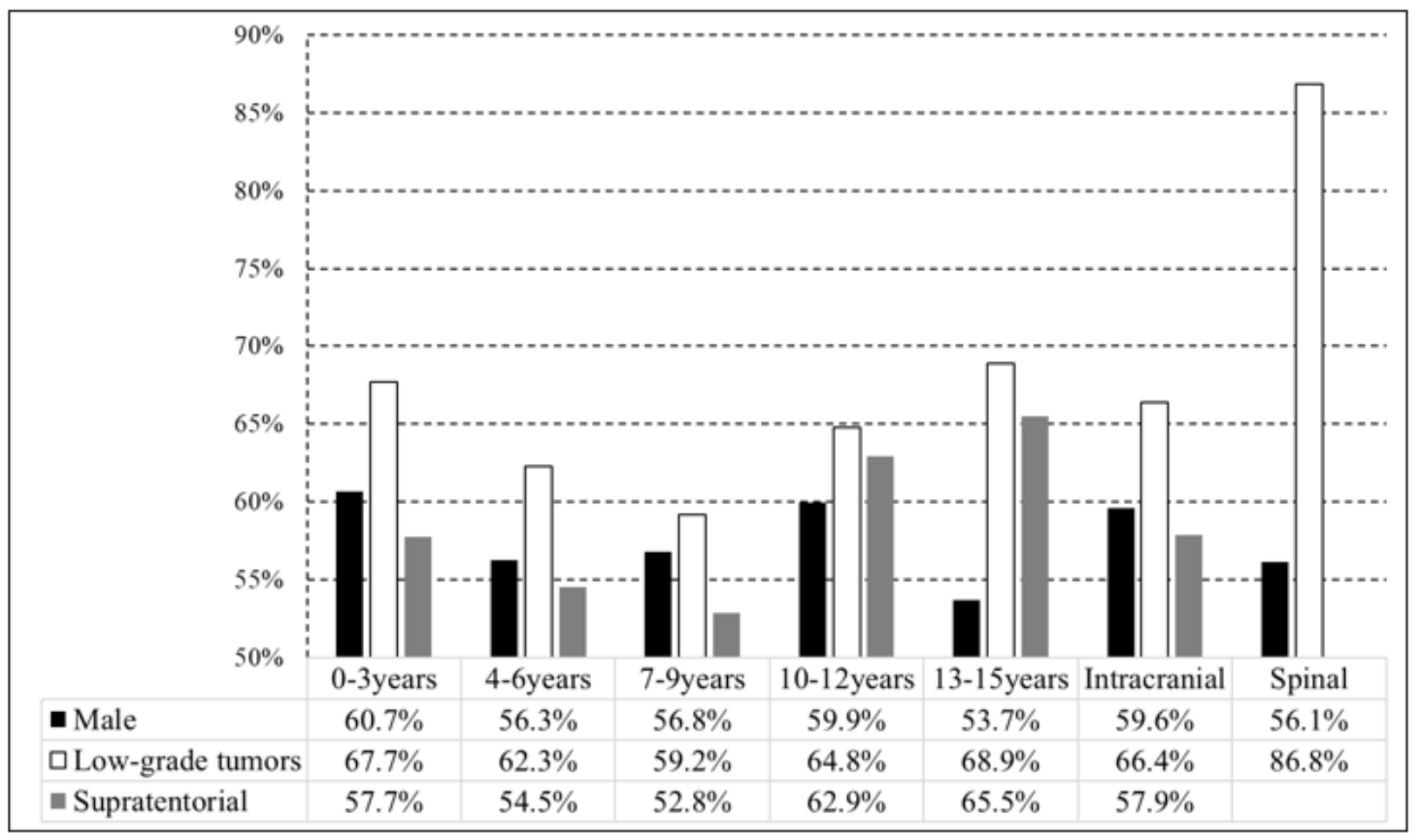

\section{Figure 1}

The percentage of male patients, supratentorial location and low-grade tumors of neurological neoplasms 
Diffuse astrocytic and oligodendroglial tumors

Other astrocytic tumors

Ependymal tumors

Other gliomas

Choroid plexus tumors

Neuronal and mixed neutonal-glial tumors

Tumors of the pineal region

Embryonal tumors

Tumors of cranial and paraspinal nerves

Meningiomas

Mesenchymal, non-meningothelial tumors

Lymphomas

Histiocytic tumors

Germ cell tumors

2010

$13.18 \%$

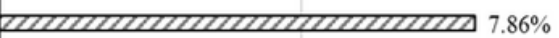

$0.03 \%$

$3.16 \%$

$10.96 \%$

$1.14 \%$

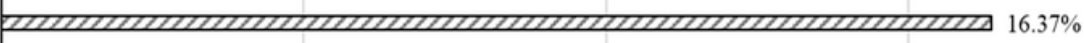

$1.34 \%$

$1.96 \%$

$1.08 \%$

$0.26 \%$

$0.07 \%$

$7.86 \%$

Tumors of the sellar region

2015 $15.39 \%$

$\begin{array}{lll}0 \% & 5 \% & 10 \%\end{array}$

$15 \%$

\section{Figure 2}

The percentage of various types of childhood intracranial tumors according to the $2016 \mathrm{WHO}$ classification of central nervous system tumors 


\begin{tabular}{|c|c|c|c|}
\hline \multirow{2}{*}{ Histological types } & \multicolumn{2}{|c|}{ Intracranial } & \multirow{2}{*}{ Spinal } \\
\hline & Supratentorial & Infratentorial & \\
\hline Diffuse astrocytoma & $118(3.71)$ & $184(5.79)$ & $6(0.19)$ \\
\hline Anaplastic astrocytoma & $17(0.53)$ & $23(0.72)$ & $2(0.06)$ \\
\hline Glioblastoma & $61(1.92)$ & $35(1.10)$ & $1(0.03)$ \\
\hline Diffuse midline glioma, H3K27M-mutant & $4(0.13)$ & $30(0.94)$ & $3(0.09)$ \\
\hline Olig odendroglioma & $9(0.28)$ & $2(0.06)$ & - \\
\hline Anaplastic oligodendroglioma & $7(0.22)$ & $4(0.13)$ & - \\
\hline Olig oastrocytoma & $37(1.16)$ & $29(0.91)$ & $1(0.03)$ \\
\hline Anaplastic oligoastrocytoma & $17(0.53)$ & $16(0.50)$ & - \\
\hline Pilocytic astrocytoma & $140(4.40)$ & $222(6.98)$ & $8(0.25)$ \\
\hline Subependymal giant cell astrocy toma & $28(0.88)$ & - & - \\
\hline Pleomorphic $\mathrm{x}$ anthoastrocytoma & $14(0.44)$ & - & - \\
\hline Subependymoma & $1(0.03)$ & $2(0.06)$ & - \\
\hline Myxopapillary ependymoma & - & - & $2(0.06)$ \\
\hline Ependymoma & $34(1.07)$ & $56(1.76)$ & $9(0.28)$ \\
\hline Anaplastic ependymoma & $57(1.79)$ & $91(2.86)$ & $2(0.06)$ \\
\hline Astroblastoma & $1(0.03)$ & - & - \\
\hline Choroid plexus papilloma & $53(1.67)$ & $11(0.35)$ & - \\
\hline Atypical choroid plexus papilloma & $12(0.38)$ & $3(0.09)$ & - \\
\hline Choroid plexus carcin oma & $17(0.58)$ & $1(0.03)$ & - \\
\hline Dysembryoplastic neuroepithelial tum or & $86(2.70)$ & $3(0.09)$ & - \\
\hline Gan gliocytoma & $4(0.13)$ & $3(0.09)$ & $2(0.06)$ \\
\hline Ganglioglioma & $81(2.55)$ & $19(0.60)$ & $5(0.16)$ \\
\hline Anaplastic ganglioglioma & - & $1(0.03)$ & $1(0.03)$ \\
\hline Dysplastic cerebellar gan gliocytoma (Lhermitte- Duclos disease) & - & $2(0.06)$ & - \\
\hline Desmoplastic infantile astrocytoma and ganglioglioma & $8(0.25)$ & $3(0.09)$ & - \\
\hline Papillary glioneuronal tumour & $39(1.23)$ & $18(0.57)$ & - \\
\hline Central neurocytoma & $6(0.19)$ & - & - \\
\hline Ex traventricular neurocytoma & $5(0.16)$ & $1(0.03)$ & - \\
\hline Parag angliom a & $1(0.03)$ & - & - \\
\hline Mix ed glioneuronal tumour & $39(1.23)$ & $17(0.53)$ & $1(0.03)$ \\
\hline Pineocytoma & $2(0.06)$ & - & - \\
\hline Pineal parenchymal tumour of intermediate differentiation & $8(0.25)$ & - & - \\
\hline Pineoblastoma & $22(0.69)$ & - & - \\
\hline Papillary tumour of the pineal region & $3(0.09)$ & - & - \\
\hline Medulloblastoma & - & $437(13.74)$ & - \\
\hline Embryonal tum our with multilayered rosettes & $11(0.35)$ & $3(0.09)$ & $1(0.03)$ \\
\hline Medulloepithelioma & - & $1(0.03)$ & - \\
\hline CNS neuroblastoma & $2(0.06)$ & $1(0.03)$ & - \\
\hline CNS embryonal tumour, NOS & $28(0.88)$ & $5(0.16)$ & $2(0.06)$ \\
\hline Atypical teratoid/rhabdoid tumuour & $8(0.25)$ & $5(0.16)$ & $1(0.03)$ \\
\hline CNS embryonal tumour with rhabdoid features & $1(0.03)$ & - & - \\
\hline Neurofibroma & $1(0.03)$ & $2(0.06)$ & $6(0.19)$ \\
\hline Perineurioma & $8(0.25)$ & $30(0.94)$ & $20(0.63)$ \\
\hline Meningioma & $59(1.86)$ & $1(0.03)$ & $6(0.19)$ \\
\hline Mesenchymal, non-meningothelial tumors & $11(0.35)$ & $22(0.69)$ & $20(0.63)$ \\
\hline Lymphomas & $6(0.19)$ & $2(0.06)$ & $1(0.03)$ \\
\hline Langerhans cell histiocytosis & $2(0.06)$ & - & - \\
\hline Germinoma & $35(1.10)$ & $2(0.06)$ & - \\
\hline Non-germin oma germ cell tumors & $199(6.26)$ & $5(0.16)$ & $14(0.44)$ \\
\hline Craniopharyngioma & $472(14.84)$ & - & - \\
\hline
\end{tabular}

\section{Figure 3}

The histological types and frequency of intracranial (including supratentorial and infratentorial) and spinal tumors [ $\mathrm{n}, \mathrm{n}(\%)$ ] 